\title{
The social and ethical impacts of artificial intelligence in agriculture: mapping the agricultural Al literature
}

\author{
Mark Ryan $^{1}$ (D)
}

Received: 6 August 2021 / Accepted: 8 December 2021

(c) The Author(s) 2022

\begin{abstract}
This paper will examine the social and ethical impacts of using artificial intelligence (AI) in the agricultural sector. It will identify what are some of the most prevalent challenges and impacts identified in the literature, how this correlates with those discussed in the domain of AI ethics, and are being implemented into AI ethics guidelines. This will be achieved by examining published articles and conference proceedings that focus on societal or ethical impacts of AI in the agri-food sector, through a thematic analysis of the literature. The thematic analysis will be divided based on the classifications outlined through 11 overarching principles, from an established lexicon (transparency, justice and fairness, non-maleficence, responsibility, privacy, beneficence, freedom and autonomy, trust, dignity, sustainability, and solidarity). While research on AI agriculture is still relatively new, this paper aims to map the debate and illustrate what the literature says in the context of social and ethical impacts. It aim is to analyse these impacts, based on these 11 principles. This research will contrast which impacts are not being discussed in agricultural AI and which issues are not being discussed in AI ethics guidelines, but which are discussed in relation to agricultural AI. The aim of this is to identify gaps within the agricultural literature, and gaps in AI ethics guidelines, that may need to be addressed.
\end{abstract}

Keywords AI ethics $\cdot$ Artificial intelligence $\cdot$ Digital farming $\cdot$ Agricultural robots

\section{Introduction}

Artificial intelligence (AI) is a term that is frequently being used, often with a degree of confusion about what it means. In popular culture, we are met with visions of Skynet, terminator-style scenarios of when AI goes wrong, or utopian futures where AI takes away all of the dirty, dangerous, and dull jobs that we are faced with. These are the most extreme versions of the tale, with many film-makers, authors, and scientists, providing insights of the in-between, and more likely, nuanced versions of AI-those that provide benefits but also raise a number of concerns, challenges, and issues that we must address head-on. Questions about the future of AI are certainly not limited. In fact, an entire field (AI ethics) has emerged to tackle these issues.

Mark Ryan

mark.ryan@wur.nl

1 Wageningen University \& Research, Wageningen Economic Research, Droevendaalsesteeg 4, 6708 PB Wageningen, The Netherlands
While there are many social and ethical issues that will be relevant for all applications of AI, there are also many unique and varied impacts and concerns for different domains, types of AI being used, and applications of AI. For example, the use of AI to detect cancerous tumours will have many health-related impacts, responsibility-allocation issues, and decision-making concerns, and will be somewhat different from the impacts faced in areas, such as logistics or marketing, for example. Therefore, the work being conducted in AI ethics is significant, but it is also important to evaluate the nuances and challenges that are unique to different domains and sectors.

This paper will examine one of those domains, namely agriculture, to identify what are some of the most prevalent challenges and impacts identified in the literature, how this correlates with those discussed in the domain of AI ethics, and are being implemented into AI ethics guidelines. Agricultural AI refers to both AI software, which often provides information, recommendations, and data; and also, AI robots. The former type of AI involves retrieving an abundance of data from the farm, local climate, data 
from machinery, and so forth, and integration AI methods (machine learning, deep learning, and reinforcement learning) to forecast and prescribe recommendations for the farmer about when to seed, harvest, and sell their crops; the health and behaviour patterns of their livestock; and image recognition to detect plant disease (to list only a few examples). Whereas, AI robots work (relatively) autonomously on the farm to harvest fruit and vegetables (such as peppers in the SWEEPER project), garden, scrape manure (LELY Robot), weeding robots (the Weed Wacker robot), drones to spray weeds and pesticides (ecorobotix), and robots that hoe and harvest (NAIO technologies' robots), and to measure crop health (agribotix) (examples taken from (Ryan et al. 2021). ${ }^{1}$

This paper will examine published articles and conference proceedings that focus on societal or ethical impacts of $\mathrm{AI}$ in the agri-food sector, through a thematic analysis of the literature. The thematic analysis will be divided based on the classifications outlined in Jobin et al. (2019). They provide 11 overarching principles, which they found in their analysis of $84 \mathrm{AI}$ ethics guidelines. While research on AI agriculture is still relatively new, this paper aims to map the debate and illustrate what the literature says in the context of social and ethical impacts. It aims to analyse these impacts, based on the principles outlined in Jobin et al. (2019). This research will contrast which impacts are not being discussed in agricultural $\mathrm{AI}$ and which issues are not being discussed in AI ethics guidelines, but which are discussed in relation to agricultural AI. The aim of this is to identify gaps within the agricultural literature, and gaps in AI ethics guidelines, that may need to be addressed.

\section{Artificial intelligence, ethics, and agriculture}

Artificial intelligence (AI) is often a contested term in the literature, but it is typically associated with the many techniques employed within it (e.g., deep learning, reinforcement learning, or machine-learning). Many things get bundled into the category of $\mathrm{AI}$ as it is has become a very profitable industry. A recent study showed that over $40 \%$ of "AI startups" are not in fact even using AI (Vincent 2019). Companies want to jump on the bandwagon and there is a lot of conflation of AI with other technologies that are not necessarily AI. Therefore, outlining, defining, and distinguishing AI, is an important first step for analysing its impact in the agricultural sector.

\footnotetext{
${ }^{1}$ Some of these projects can be found at the following links: http:// www.sweeper-robot.eu/; http://trimbot2020.webhosting.rug.nl/; https://www.lely.com/solutions/housing-and-caring/discovery-colle ctor/.
}

AI first emerged in the 1950s through a group of computer scientists working in the field, developing very primitive (compared to modern standards) neural networks. Their early work in the field spawned what is nowadays called AI. The term was first coined at a conference at Dartmouth College in 1956, by John McCarthy (MIT). AI is typically understood to be a system, or systems, that can display intelligent behaviour through the analysis of their environment and take actions, with relative autonomy, to achieve specific goals (European Commission 2018). AI can perform many complex tasks that were previously done by humans, 'such as image recognition (vision), speech recognition (hearing), and natural language generation (speaking). AI is artificial mimicry of tasks and functions that would otherwise require human intelligence' (Ryan 2020).

AI can typically take on the form of software/programming or embodied in physical structures (such as humanoid robots, drones, self-driving vehicles, or medical devices). However, not all robots have AI. There are many non-AI robots that may be hand-controlled by a human or have a very limited range of autonomous skills. These are often referred to as industrial robots within the robotics community, as opposed to advanced robots, which use AI. The use of AI robots within popular culture often gives the impression that AI has reached a level where it can feel, understand, and rationalise in the same way as human beings can, or at least, that this is something that will happen soon. This is one reason that the analysis of robot rights, superintelligence, and AI singularity, have become so popularised within the literature. However, many of these topics are still very speculative and some claim that they veer away from more pressing, urgent, matters related to AI (Birhane and van Dijk 2020). For example, human rights infringements caused by AI use, harmful biases based on discriminatory data, analysis, and interpretation; predictive policing algorithms to over-police historically disadvantaged areas; and the use of AI in warfare, which further dehumanises combatants and bystanders.

General AI refers to AI that has the ability to perform a wide range of tasks that would have normally been done by human beings. While narrow AI focuses on specific and limited tasks, general AI has the capacity to perform a wide diversity of these tasks and has human-level (or beyond) intelligence. General AI does not (yet) exist, so it is still very speculative, and will not be discussed in this paper (Russell 2021). The main focus is to identify ethical and social issues pertaining to narrow AI found in the agricultural sector. However, first, it is important to give a bit of background about the types of social and ethical impacts being discussed in the AI literature and how these may relate to the agricultural sector. 


\subsection{Artificial intelligence applications in agriculture}

As discussed in the previous section, sometimes it may be easier to split AI into two types of applications: AI software and AI robots. AI software is required for the functioning of AI robots, but AI robots are not (necessarily) required for the functioning of AI software. The purpose of this division is to clearly demonstrate the two most significant types of AI applications within the agricultural sector. ${ }^{2}$

The use of (non-AI) robots in agriculture has been around for quite some time, with robot milking being used for almost two decades now. However, the use of agricultural AI robots is still relatively new. Agricultural robots are being used in a wide variety of ways: crop scouting, pest and weed control, harvesting, spraying, pruning, milking, phenotyping, and sorting (Shamshiri et al. 2018). These types of robots must be able to adapt to their environment and navigate their way through farmland. Most AI robots are still in the early stages of development, with many taking shape in testing facilities, research projects, and research centres. There are very few that have reached a commercial scale, and most cannot compete with the speed of their human counterparts to fulfil their activities (e.g., weeding and harvesting robots) (Shamshiri et al. 2018).

AI is also being deployed in other types of robots, such as drones and self-driving tractors. Drones are being used to spray fields with water, pesticides, herbicides, and so forth. They are also being used to take aerial photographs and images of the farm and its surrounds. Drones are providing insights and mapping of the farm, which would not have been otherwise possible. Whereas self-driving tractors hold great potential for farmers to be able to do other activities. However, self-driving tractors are still in very early stages of development and have not been deployed in a commercial setting, yet. Similarly, to other self-driving vehicles, there are a lot of safety and security factors that must be considered before they can be integrated.

In addition, AI is also being used in apps, recommendation systems, and software. Image recognition is allowing

\footnotetext{
${ }^{2}$ Of course, AI software benefits from the use of hardware on the farm, through the uses of sensors and monitors, which retrieve data to train AI; however, typically, these sensors are devoid of AI. While AI robots may be integrated with, and use, sensors; they are much more integrated within the overall functioning of the robot, as opposed to the much more distant and detached sensors used for improving AI software. Of course, this distinction is somewhat arbitrary, and one could also make the claim that non-AI sensors that are used to improve AI software should be considered part of the AI system as a whole; that the distance or level of detachment should not matter. This paper will take the former approach, but does not rule out the latter option. Regardless, this will not impact the position of the paper or the analysis of discussions of agricultural AI found in the literature.
}

farmers to determine the health, or illness, of a particular plant or crop and to provide recommendations about what they should do (Ryan 2019). Some AI maps the life-stages and growth of plants, how this will change throughout its cycle, and what actions should be taken by the farmer (Ryan 2019). AI is replacing many of the tasks typically designated to the agronomists and is also allowing farmers to streamline their documentation and administrative burdens. Companies, such as BASF, Monsanto, Bayer, Pioneer, and John Deere, are using the data retrieved from farms to provide tailored insights and recommendations to farmers, with the assistance of AI technologies. However, this is not new and is typically referred to as 'precision agriculture', which is 'a management strategy that gathers, processes and analyzes temporal, spatial and individual data and combines it with other information to support management decisions according to estimated variability for improved resource use efficiency, productivity, quality, profitability and sustainability of agricultural production' (taken from the Precision Agriculture journal: https://www.springer.com/journal/11119). However, what is new about agricultural AI are the tools and types of innovation being used to achieve these goals. Overall, AI is providing insights into what a farmer should do, when, how, and to what extent, on their farm. Thus, it is important to bear in mind these different applications of AI in agriculture for the purpose of this paper.

\section{Methodology}

The purpose of this paper is to evaluate current ethical and social challenges of $\mathrm{AI}$ in the agricultural sector. Ethical and social discussions stem back over 2000 years, with a long heritage of analysing what it means to live a good life, how to live a good life, and why we should even care about it to begin with. In its broadest interpretation, it means distinguishing between right and wrong, and determining ways one should behave in particular circumstances and contexts. It has materialised in many different frameworks, such as identifying what a virtuous person would do in a particular situation (virtue ethics), following distinctive ethical rules of what is right and wrong (deontology), or acting based on what will result in the best outcome for the most amount of people (utilitarianism).

This paper will not adopt any single ethical framework to guide this research, instead focusing on what are the most prevalent and significant issues, values, and morals, being discussed in the AI agricultural literature. It will examine the current landscape of ethical and social impacts of AI and will offer insights into the most commonly discussed principles in the debate, while analysing how they may be different from other industries. It will do this using the 11 thematic principles outlined in Jobin et al. (2019) (see 
Table 1 Principles and constituent ethical issues or guidance of 84 ethics guidelines, as outlined by Jobin et al. (2019), and visualised by Ryan and Stahl (2020)

\begin{tabular}{|c|c|c|c|c|}
\hline \multirow{2}{*}{$\begin{array}{l}\text { Principle } \\
\text { Transparency }\end{array}$} & \multicolumn{4}{|l|}{ Constituent ethical issues or guidance } \\
\hline & Transparency & Explainability & Explicability & Understandability \\
\hline & Interpretability & Communication & Disclosure & Showing \\
\hline \multirow[t]{4}{*}{ Justice and fairness } & Justice & Fairness & Consistency & Inclusion \\
\hline & Equality & Equity & Non-bias & Non-discrimination \\
\hline & Diversity & Plurality & Accessibility & Reversibility \\
\hline & Remedy & Redress & Challenge & Access and distribution \\
\hline \multirow[t]{2}{*}{ Non-maleficence } & Non-maleficence & Security & Safety & \\
\hline & Protection non-subversion & Precaution & Prevention & Integrity \\
\hline Responsibility & Responsibility & Accountability & Liability & Acting with integrity \\
\hline Privacy & Privacy & Personal or private information & & \\
\hline Beneficence & Benefits social good & Beneficence common good & Well-being & Peace \\
\hline Freedom and autonomy & Freedom & Autonomy & Consent & Choice \\
\hline Trust & Self-determination trustworthiness & Liberty & Empowerment & \\
\hline Sustainability dignity & Sustainability dignity & Environment (nature) & Energy & Resources (energy) \\
\hline Solidarity & Solidarity & Social security & Cohesion & \\
\hline
\end{tabular}

Table 1$).^{3}$ Choosing any specific lexicon will have an element of arbitrariness attached, but this lexicon was chosen because it represents the most comprehensive mapping of AI ethics guidelines at present, and thus, works as a way to represent most of the principles that are important around the topic of AI. 'Eleven overarching ethical values and principles have emerged from our content analysis. These are, by frequency of the number of sources in which they were featured: transparency, justice and fairness, non-maleficence, responsibility, privacy, beneficence, freedom and autonomy, trust, dignity, sustainability, and solidarity' (Jobin et al. 2019, p. 391) (see Table 1).

It must be noted here that the 11 principles outlined in Jobin et al. (2019), are simply used to structure the topics and principles that arise within the systematic literature analysis. The analysis of ethical themes outlined in Jobin et al. (2019) will be identified, but the literature analysis will not be confined to these themes, as will become evident in the discussion section of this paper, where additional ethical topics are discovered in the literature (that do not necessarily fit within the 11 categories of Jobin et al. 2019). While the Jobin paper provides an excellent framework to identify and map ethical principles within a literature analysis, it

\footnotetext{
3 While we are aware that some of the topics outlined by Jobin, Ienca, and Vayena 2019, and which will be used in this paper, could be argued as not being explicitly social or ethical topics, we believe that this is one of the best breakdowns of themes being discussed in relation to social and ethical impacts of AI. In any such comprising of lists, one may be overly critical, but we are aware that there will always be an element of arbitrariness and tendentiousness with such a compiling or classification (Sparrow and Howard, 2020).
}

was limited in the fact that some of the themes emerging within the agricultural AI ethics literature did not accurately fit within these categorisations.

For the purpose of this paper, we conducted a systematic literature review of available English-language texts covering the broad topic of ethical and social impacts of agricultural AI. Systematic literature reviews aim to provide insights and added information about the current state of research in a particular area and to map correlations, similarities, and divergences within the literature. It is also used to identify potential gaps in that literature, which require further research. To identify the ethical and social dimensions of agricultural AI, a systematic review of the literature was conducted to identify papers, books, chapters, and conference proceedings.

The methodology employed to find relevant literature was through Scopus and Google Scholar searches. First, the following search query was used in Scopus: "TITLE-ABSKEY (ethics OR society OR moral OR norm OR ethical AND agriculture OR farm OR farms OR farming OR agricultural AND ai OR artificial AND intelligence OR robot OR robots OR robotic)". This query retrieved 269 results. The screening step involved analysis of the titles, abstracts, and scanning the texts for relevance for the focus of our research. However, out of this list of 269 articles, 261 articles were excluded because they met the exclusion criteria that was created for the purpose of this research. The types of research that were excluded were:

- Research that does not give specific attention to ethical impacts of AI

- Research that only focuses on non-AI robotic technology 
- Research that only focuses on ethical aspects of AI, but not related to agriculture

- Research that only focuses on non-AI agricultural technology

- Research that only focuses on technical challenges related to agricultural AI

For example, articles, such as (Vik et al. 2019) and (Driessen and Heutinck 2015), focused on impacts of using non-AI milking robots, which would deem it unsuitable for the purpose of this paper. While articles such as (Bronson 2019) do not specifically focus on AI, but more broadly the impacts of digitalisation in farming and does not give specific reference to AI. Many of the articles that were screened out were in disciplines far removed from the agricultural sector, such as nursing, mathematics, and psychology. These were removed during the title and abstract screening, rather than conducted separately, because of their clear lack of relevance for the literature analysis.

To supplement these articles, a Google Scholar search was conducted, with the same search criteria outlined earlier, but using Google Scholar's search system, to find additional articles that were not uncovered in the Scopus search. Another 13 articles were discovered using this process, bringing the overall number to 21 articles. The ROSES protocol was used to conduct this literature search review (see Fig. 1).

Not all of the articles had an even spread of social and ethical topics covered within them. Some of the papers had far more references to social and ethical themes than others. This is because of the diversity of articles and the range of disciplines analysed. For example, some of the papers were in social science journals, but there were also some in engineering and computer science journals (Aggarwal and Singh 2021; Albiero 2019; Krishnan and Swarna 2020; Marinoudi et al. 2019; Shamshiri et al. 2018). Not surprisingly, the articles by social scientists and ethicists had the greatest emphasis on social and ethical themes, which can be illustrated in Table 2 (Gardezi and Stock 2021; Rose et al. 2021; Ryan 2020, 2019; Sparrow and Howard 2020; Stock and Gardezi 2021). This is understandable as much of the focus of these papers is directed towards ethical and social issues, while the papers found in the engineering and computer science journals were more directed towards technical challenges, with ethical issues often having importance, but to a lesser degree. This difference can be seen in Table 2.

Table 2 also represents how many times the 11 ethical principles (as outlined by Jobin et al. 2019) were found or discussed within each of these papers. Another striking insight from Fig. 1 is the newness of the field. Out of the 21 articles analysed, one was from 2018, six from 2019, six from 2020, and eight from 2021. This emphasises the novelty of the field and the importance of this research to classify and map the social and ethical impacts of agricultural AI.

The 21 articles were analysed using a thematic analysis methodology (Aronson 1995; Braun and Clarke 2006). The contents of the articles were classified and analysed by the most significant ethical themes, principles, and values, discussed within AI ethics guidelines (Jobin et al. 2019; Ryan and Stahl 2020). The Jobin et al. (2019) article provided a rigorous examination of $84 \mathrm{AI}$ ethics guidelines and found overlapping themes within theme, which consisted of 11 overarching principles, and 61 constituent topics which fall under the rubric of the principles (see Table 1 earlier).

The ethical principles found within the 21 articles were categorised using the free trial version of MAXQDA 2020 data analytics software to provide an initial quantitative overview of the principles within the 21 papers. This was done by both lexical searches for the phrases within the 11 categories and 61 constituent topics, and contextual analysis of the texts to identify if these issues were implicitly discussed. The same 11 overarching principles and subsequent 61 ethical themes (outlined in Jobin et al. 2019) were used for the lexical search queries and coding (Table 3).

\section{Results}

The classification outlined by Jobin et al. (2019) was used to code our research because it is the most comprehensive mapping of social and ethical themes within the AI ethics debate. The aim of this paper is to begin by mapping the most discussed principles in the agricultural literature, and second, the content of these papers in relation to these respective thematic areas. While Jobin et al. (2019) is the most comprehensive outline of ethical and social issues within the AI debate, it is also important to identify if the current ethics guidelines have a corresponding importance for the agricultural sector or if there are different requirements within this sector.

This is important because it helps us to identify if the values that are discussed in AI ethics guidelines are representative of what the agricultural AI literature finds important. This paper also aims to illustrate gaps that are not being discussed in the agricultural AI debate, but are elsewhere (namely, the $84 \mathrm{AI}$ ethics guidelines analysed by Jobin et al. 2019). This comparison can be seen in Fig. 2 below, with the number of times the 11 ethical principles appeared in the 21 articles (centre column), compared to the Jobin et al. (2019) paper, which lists the number of times those ethical principles emerge within the $84 \mathrm{AI}$ ethics guidelines that they evaluated (right column).

An interesting insight is that both the literature review and the AI ethics guidelines place less emphasis on principles, such as dignity and solidarity. This may be because 
ROSES Flow Diagram for Systematic Reviews. Version 1.0

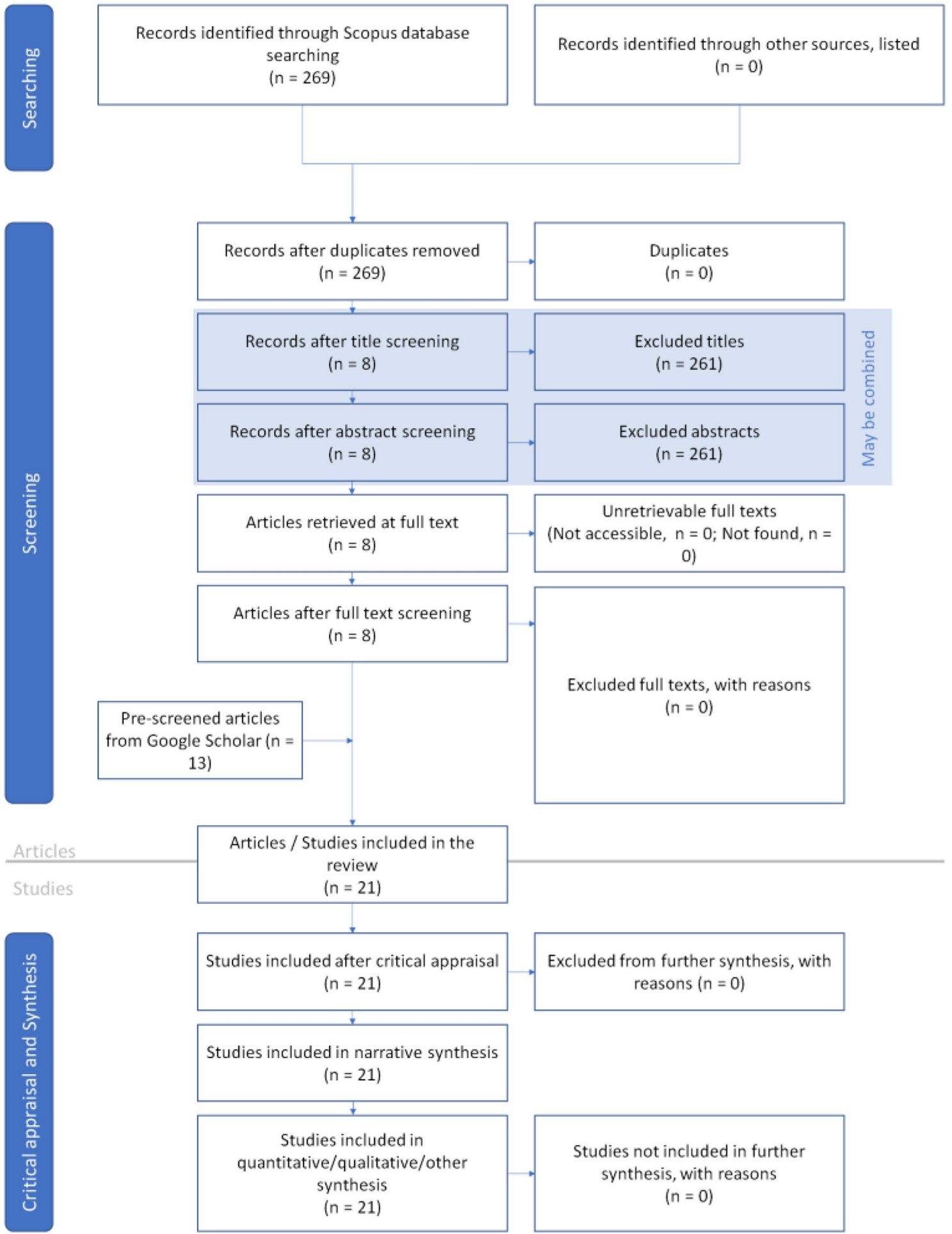

Fig. 1 ROSES Flow Diagram for Systematic Reviews (Haddaway NR, Macura B, Whaley P, and Pullin AS. 2017. ROSES flow diagram for systematic reviews. Version 1.0. https://doi.org/10.6084/m9.figshare.5897389) 
they are not so relevant for all applications and uses of AI (such as agriculture), so receive less attention as other principles, such as beneficence and non-maleficence, which receive a similar amount of attention in both the agricultural AI literature and the AI ethics guidelines. There are many other principles that are fairly congruent between the two, such as privacy and responsibility.

Table 2 Articles (listed by author, title, date) with the number of times the list of principles outlined in Jobin et al. 2019 appear in the text

\begin{tabular}{ll}
\hline Article reference & $\begin{array}{l}\text { Times the 11 } \\
\text { principles were } \\
\text { referenced }\end{array}$ \\
\hline (Aggarwal and Singh 2021) & 12 \\
(Albiero 2019) & 2 \\
(Bogomolov et al. 2021) & 59 \\
(Camaréna 2020) & 137 \\
(Carolan 2020) & 20 \\
(Gardezi and Stock 2021) & 219 \\
(Klerkx et al. 2019) & 93 \\
(Krishnan and Swarna 2020) & 26 \\
(Marinoudi et al. 2019) & 19 \\
(Pylianidis et al. 2021) & 92 \\
(Rodzalan et al. 2020) & 48 \\
(Rose and Chilvers 2018) & 43 \\
(Rose et al. 2021) & 46 \\
(R Shamshiri et al. 2018) & 37 \\
(Ruiz-Real et al. 2020) & 21 \\
(Ryan 2020) & 58 \\
(Ryan 2019) & 122 \\
(Sparrow and Howard 2020) & 88 \\
(Stock and Gardezi 2021) & 77 \\
(Vasconez et al. 2019) & 83 \\
(Wang et al. 2021) & 108 \\
\hline
\end{tabular}

Table 3 The 11 overarching principles identified in our research (left) beside the number of times those searches appeared in the $84 \mathrm{AI}$ ethics guidelines that Jobin et al. 2019 analysed (right)
What is perhaps more interesting is the striking differences at both extremes of the two Figures. Sustainability is the most discussed principle within agricultural AI, but is one of the least significant principles found within the AI ethics guidelines. This is very significant and may suggest a need to give a greater emphasis on sustainability within AI debates. In addition, trust has been well-documented as being an important principle within the agricultural sector, specifically, in relation to data-sharing and deploying emerging technologies on the farm (Carolan 2006; Gardezi and Stock 2021; Jayashankar et al. 2018; van der Burg et al. 2020; Wiseman and Sanderson 2018). The value of trust is very important, and the adoption of AI within the sector brings further meaning of how trust will be created and reinforced. Whereas, it received less importance within AI ethics guidelines (Jobin et al. 2019). However, this is changing since the 2019 High-level Expert Groups' 'Ethics Guidelines for Trustworthy Artificial Intelligence' (HLEG 2019). Since then, there have been numerous articles and reports emphasising the importance of trust within AI (AI HLEG 2020; Floridi 2019; Lewis and Moorkens 2020; Malta Digital Innovation Authority 2019; Zicari 2021).

One glaring omission from the agricultural $\mathrm{AI}$ literature is the importance of transparency. While it is barely mentioned in the agricultural AI documents, it is seen as the most significant principle within AI ethics guidelines. There are no self-evident reasons for the lack of analysis of transparency of AI in agriculture, so this appears to be a significant gap, rather than an omission because of its lack of importance. This points to the need for more research to be conducted on transparency during the development, deployment, and use of AI, in the agricultural sector.

The following subsections will outline the specific content found within the 21 papers analysed, using the thematic division of the 11 overarching principles discussed earlier. It will be shown that there is a strong degree of overlap within the literature on certain issues and themes, but they varied in how they were discussed (e.g., some were descriptive of

\begin{tabular}{lll}
\hline Ethical Principles & $\begin{array}{l}\text { No. of times found in the 21 } \\
\text { papers analysed for this paper }\end{array}$ & $\begin{array}{l}\text { No. of times found in the 84 AI ethics } \\
\text { guidelines analysed by Jobin et al. } \\
2019\end{array}$ \\
\hline Sustainability & 595 & 14 \\
Non-maleficence & 228 & 60 \\
Trust & 209 & 28 \\
Beneficence & 122 & 41 \\
Freedom and Autonomy & 68 & 34 \\
Privacy & 66 & 47 \\
Justice and Fairness & 53 & 68 \\
Responsibility & 47 & 60 \\
Transparency & 19 & 73 \\
Solidarity & 2 & 6 \\
Dignity & 1 & 13 \\
\hline
\end{tabular}


issues, while others were more orientated towards recommendations). The following 11 subsections (based on Jobin et al. 2019) will highlight the social and ethical content being discussed in the agricultural AI literature.

\subsection{Sustainability}

One of the themes that kept arising within the literature was sustainability (Albiero 2019; Bogomolov et al. 2021; Krishnan and Swarna 2020; Ruiz-Real et al. 2020; Vasconez et al. 2019). Sustainability is a very important concern for farmers and the agricultural sector, and deploying AI must be able to not only meet current sustainability achievements, but surpass them (Ryan 2019). Robots, sensors, and drones, hold the potential to leak fluid, toxic chemicals, and emit fumes, on the farm, as well as the highly burdensome ecological impact required to produce these machines in the first place (Ryan 2019). For example, AI may cause 'surface water run-off, encroachment on habitats, or general pollution to the surrounding area' (Ryan 2019, p.12).

There is a concern that the increased technologization of agriculture will lead to plants, crops, and livestock being modified to better accommodate these technologies (instead of the other way around). Because of the nature of AI robots (they often find it difficult to reach certain plants and crops, or damage them when using their robotic arms to retrieve them), there have been discussions that plants should be genetically modified to better accommodate the robot (Sparrow and Howard 2020). In addition to this, the use of AI may also lead to less sustainable practices and outcomes as a whole: 'It is, for instance, possible that application of pesticides might actually increase as the costs of applying them are lowered or that more powerful-and perhaps dangerous-pesticides might be used once human beings were no longer involved in their application. If human workers are replaced by heavier robots this might compound existing problems arising from soil compaction due to the use of heavy machinery in farming' (Carolan 2020, p. 821).

\subsection{Non-maleficence}

Health and safety of those on the farm should be ensured when deploying and using agricultural AI (Vasconez et al. 2019). Some cite that the use of AI may help reduce exposure to toxic chemicals and pesticides, protecting protect farmers from harmful health effects on the farm (Rodzalan et al. 2020). In addition, AI may reduce accidents and physical harms to the farmer. As most accidents during human-machine interaction are caused by human error (Vasconez et al. 2019), a greater level of machine automation may reduce this. This is a point that has been expressed as an advantage of using more autonomous machinery namely that it will lead to less accidents and safer farms (Ryan 2019).

The risk of using AI within the agricultural sector is sometimes quite high as it is dealing with livestock, wild animals, and living ecosystems (Pylianidis et al. 2021). Ensuring that no harm is done during the deployment of AI is a significant challenge that must be addressed within the field. Some farmers have reported that they believe the use of AI robots and drones may lead to more harmful and riskier farming, particularly towards the natural world, because there is less fear of harms towards humans (Gardezi and Stock 2021). An additional safety concern that was highlighted by Carolan (2020) is farms that 'are highly reliant on automation and robotics will become correspondingly more vulnerable to hacking, sabotage, and corporate espionage' (Carolan 2020, p. 826).

\subsection{Trust}

Data derived from AI may improve transparency and promote trust, but it is also difficult for humans to trust AI because it lacks emotive states (Rose et al. 2021). However, building trust within the agricultural sector is quite challenging (Pylianidis et al. 2021). Trust is difficult to ensure when autonomous machines are deployed on the farm, collecting data that is sent back to the agribusiness, which is largely unknown to the farmer (Stock and Gardezi 2021). Despite the low level of references to trust among this particular collection of papers, trust is a widely discussed topic within digital agriculture, more generally (Brewer et al. 2021; Jakku et al. 2019; Jayashankar et al. 2018; van der Burg et al. 2020; Wiseman and Sanderson 2018). This indicates that trust is probably an important issue for agricultural AI, as well, but just not enough research has been done on the topic yet.

\subsection{Beneficence}

Some papers criticised 'Big Tech' (Camaréna 2020) or the power of agribusinesses (Ryan 2020), stating that farmers should be given greater inclusion within the decision-making process. Essentially, 'the co-designed scenarios of the future need to be reclaimed by the commons and informed by expertise in the different fields, including the engineering and AI experts who can jointly define the goals for the use of AI in such a vital field (food systems). As we have seen, critical to future sustainable food systems is the need for co-creating solutions, including stakeholders across all the disciplines as well as government and communities' (Camaréna 2020, p. 12).

There is a pressure on farmers to integrate AI for the common good. Farmers who adopt innovative technologies are viewed as having foresight, doing their part for society, and acting in a morally praiseworthy manner (Gardezi and Stock 
2021). Those who do not are viewed as acting carelessly, selfishly, and defined as 'laggards'. Farmers are boxed into certain groups, those who are forward-looking and progressive, or those who want to remain in the past. Farmers are put in a difficult position, as they must modernise or be left behind—both socially and economically.

\subsection{Freedom and autonomy}

Because of the legalistic and technical jargon used by agribusinesses and tech providers, farmers may not understand the legal agreements that they are entering into when using AI (Ryan 2019). This has led to a number of issues in recent years, with some large agribusinesses gaining greater control over farms. Some companies prohibit farmers from repairing or 'tampering' the AI hardware, which they purchase and use on their farm, stripping farmers of the freedom to interact with their own tractors, tools, and hardware (Ryan 2019). This has a very restrictive effect on the farmer, making them feel like a worker (for the agribusiness) on their own farm.

In addition, farmers have internalised the algorithmic authority of AI decision-making (Gardezi and Stock 2021). Their control and freedoms over their farms are given over to the AI system, which tells them the 'correct' or 'best' way to farm, because data-driven recommendations appear to come from a scientifically proven source. This may be in opposition to their own views of how to run their farm. They feel pressured into accepting these decisions, as a result of investing so much money into these technologies or from the agribusinesses who supply them (Ryan 2020).

\subsection{Privacy}

There is a concern about what types of data are retrieved by $\mathrm{AI}$ and how these data will be disseminated and used (Ryan 2019). The data retrieved, generated, and stored, from agricultural AI, raise concerns around data ownership and privacy (Wang et al. 2021). Often, farmers must trade-off their privacy for the benefits and promises of AI on their farm; with $78 \%$ of farmers agreeing that they are concerned about their data being shared and sold on by corporations (Stock and Gardezi 2021). The concern of privacy infringements, from AI, is a significant concern within the sector.

\subsection{Justice and fairness}

There is a possibility that AI will create digital divides between farms, countries, and the industry as a whole (Ryan 2020, 2019). If AI is only deployed on rich, large, monocultural farms, this seriously limits the uptake of AI by smaller farms, poorer farmers, and the Global South. Carolan (2020) noted that because of the historically low numbers of women in STEM and farming, the increased integration of technologizing the farm, may further exacerbate the number of women in agriculture. The challenge is to ensure that everyone will benefit from AI in a fair and inclusive way (Aggarwal and Singh 2021).

Another concern is that AI will bring economic benefits to agribusinesses and tech companies, but not the farmer themselves (Ryan 2020). In addition to this, there is a worry that economic benefits of AI would permit social, ethical, and environmental harms. Thus, farmers, advisors, and society, 'should be able to question and contest whether benefits to productivity should supersede social, ethical, or environmental concerns, and be able to convince innovators and policy-makers to change the directions of innovations for sustainable agriculture' (Rose and Chilvers 2018, p. 5).

\subsection{Responsibility}

Agribusinesses may use AI unscrupulously on farms, with little repercussions (Ryan 2020). They may use the data retrieved from agricultural AI to upsell products to the farmer, tie their use of AI to their seed and machinery supplies, or use these data to buy up their land for very low prices (Ryan 2020). Ryan (2020) gives the example of how Pioneer and Monsanto require their AI customers to be prior customers of their seeds. Carolan (2020) discusses how John Deere makes farmers sign a licensing agreement that they will not sue the company if there is damage to their crops, land, profit, machines, or business (Carolan 2020). If one is dependent on these companies' seeds or tractors, and their agreements become entangled with AI use, then it becomes much more difficult to severe ties with these organisations if one no longer wants their AI (Ryan 2020).

Furthermore, the onus of responsibility often shifts onto the farmer to provide the adequate environment and circumstances for the AI to work. There is pressure that the AI will fulfil its function if they are given an appropriate working environment and are implemented correctly (Stock and Gardezi 2021). The onus of responsibility is on the farmer to ensure that the AI/robot can work freely and that all obstacles (informational and physical) are removed from its path (Stock and Gardezi 2021).

\subsection{Transparency}

There is a concern about the interpretability and understandability of the data being retrieved from AI systems and if these recommendations are accurate (Ryan 2019). The consequences of inaccurate recommendations may lead to harms to the farmer, livestock ill-health, unsustainable practices, and welfare harms on the farm (Ryan 2019). 
Thus, there needs to be a greater level of education, training, and understandability of AI, within the agricultural sector to understand how these technologies work and what their limitations are (Pylianidis et al. 2021).

\subsection{Solidarity}

Solidarity was only mentioned twice in the documents. ${ }^{4}$ The lack of solidarity references in the literature is because $\mathrm{AI}$ in agriculture is relatively new and the topic of solidarity is not a theme widely discussed in AI ethics literature. For example, with the $84 \mathrm{AI}$ ethics guidelines evaluated by Jobin et al. 2019 , only six referenced solidarity. This is not to propose that it is not a potentially significant area of research, simply that it did not arise within the articles reviewed.

\subsection{Dignity}

Dignity was only mentioned once in an indirect and passing way. ${ }^{5}$ Similarly, to solidarity, dignity is often not widely discussed in the AI ethics literature, but more so in specific applications and domains of AI use, such as healthcare (Beyan et al. 2015; Milton 2021; Zardiashvili and FoschVillaronga 2020). In the context of agriculture, it was not surprising that dignity was not widely discussed. The types of AI being used in the agricultural sector do not have the same kind of dignity-infringing potential as healthcare, as they are not dealing with vulnerable patients and life-threatening diseases. The contexts are different and the harms to dignity are thus divergent. However, this is not to say that dignity is not an important topic for the agricultural sector, but it is more important in the context of labour and the meaning of work on the farm, which will be discussed in the next section.

\section{Discussion}

While the 11 principles outlined by Jobin et al. (2019) provide a helpful template to frame the social and ethical challenges within agricultural AI, it became apparent that they could not cover everything discussed within the literature. Jobin et al. (2019) based these 11 principles on the $84 \mathrm{AI}$ ethics guidelines they analysed, so the gaps that we

\footnotetext{
${ }^{4}$ One reference was in the reference section of Klerkx et al. 2019, and the second was a reference to Bourdieu in the Carolan 2020 article, which was somewhat irrelevant for this paper (see Carolan 2020, p. 188).

5 'While power is required for democratic systems to function, it can also be abused, leading to curtailments and infringements upon individuals' liberties, autonomy, dignity, and fundamental human rights' (Ryan 2020, p. 56).
}

identified not only hold potential for providing additional insights into the social and ethical dimensions of agricultural AI, but also provide insights and gaps within these guidelines. For example, there is a striking divergence between the two on the impact of AI on other species and sustainability, themes which have only recently started to receive more attention in the academic literature on AI ethics (Donhauser et al. 2020; van Wynsberghe 2021; van Wynsberghe and Donhauser 2018).

Specifically, a very important topic that arose within this research, which was not really covered within the $84 \mathrm{AI}$ ethics guidelines, was the topic of animal welfare (Ryan 2019). The anthropocentric positioning of the AI ethics guidelines is not very surprising, but perhaps, this is an area where further reflection can ensure a more ecologically sustainable approach is adopted. The agricultural AI literature discussed how AI robots may disturb, stress, and even harm, both farm animals and wildlife in the surrounding areas, and should be a factor to consider when developing these technologies (Ryan 2019). If this is not acknowledged, there is the potential that AI may be used as just another tool to further exacerbate human domination over nature (Carolan 2020).

The 11 AI ethics guidelines' principles, outlined by Jobin et al. (2019), do not contain any specific reference to employment, which seems like a strange omission, especially because one of the ethics guidelines analysed even had it in their title Work in the Age of Artificial Intelligence: Four Perspectives on the Economy, Employment, Skills and Ethics (Ministry of Economic Affairs and Employment, 2018). Despite this, employment and the future of work is a very important topic that is regularly discussed within AI ethics debate (Anderson et al. 2018; Bekken 2019; Belk 2020; Bossman 2016; CNIL 2017; Frey and Osborne 2017).

Employment came up numerous times in relation to agricultural AI, with some articles having that as their main theme (see 'Robotics and labour in agriculture. A context consideration', Marinoudi et al. 2019). In this article, Marinoudi et al. (2019) propose that the economic benefit of $\mathrm{AI}$ on the farm is the main driving force for implementing it. In Ryan (2019), interviewees from a large multinational agribusiness said that AI technology will not replace human agronomists any time soon (Ryan 2019). They reported that their $\mathrm{AI}$ is meant to be complementary to the agronomist and that many farmers want to stick to human contact for their recommendations (Ryan 2019).

Additionally, the high labour costs, the aging demographic of farmers, and future labour shortages, were all incentives for farmers to make the switch to using AI and automation on the farm (Carolan 2020; Klerkx et al. 2019; Rodzalan et al. 2020; Rose et al. 2021; Stock and Gardezi 2021). For many farmers, AI brings the opportunity for increasing welfare as they remove many of the dirty, dangerous, and dull jobs on the farm; they free up individuals' 
time to concentrate on other pursuits or allow individuals to take up alternative employment to further support the household's income (Carolan 2020; Klerkx et al. 2019; Rodzalan et al. 2020; Rose et al. 2021; Stock and Gardezi 2021).

Some interviewees in Carolan (2020) believed that the AI industry may try to encourage border controls, reduce immigration, and increase the difficulty to obtain workers' visas, because of the economic advantage that this would entailnamely, being able to sell more AI to farmers; replacing the human migrant labourer (Carolan 2020). Overall, this may result in ' $\mathrm{h}$ ] igher levels of unemployment in rural areas generated by robots, a decline in the level expertise or experience required to perform the jobs that do remain, and any increase in workplace surveillance will mean a corresponding increase in the power of employers to dictate the wages and conditions of those who are able to find work in the agricultural sector' (Carolan 2020, p. 824).

This future may also result in fewer opportunities for farmers. The skillset required to use these robots may also be limited to educated and skilled individuals, and if farms can be controlled remotely or even run autonomously, then it may impact the profession of farming altogether (Carolan 2020). However, there is also a strong degree of compatibility and complementarity with the introduction of $\mathrm{AI}$ in the agricultural sector; particularly, with non-routine cognitive tasks, such as disease detection (Marinoudi et al. 2019). Harvesting fruit and vegetables is also another example where collaboration has been shown, with humans harvesting what is missed by the AI robot (Shamshiri et al. 2018).

A concern within the literature, which was not really covered under the 11 sections, was around control and ownership of the data retrieved, stored, and used, by the AI system. One of the issues centred around a concern about data ownership and who has access and use of the data retrieved about the farmer by the AI (Ryan 2019). The concern is that farmers will no longer have control over their farms and that the agribusinesses would use the data about the farmer to sell products back to them, will benefit from their data while the farmer does not, and even the possibility that these data will be used against them by regulators and their competition (Ryan 2020, 2019).

\section{Conclusion}

This paper examined 21 published research articles that focused on the societal and ethical impacts of using AI in the agricultural sector, using a thematic analysis of the literature. The thematic analysis was derived from the work done by Jobin et al. (2019), whereby, they classified 11 overarching ethical principles that they established were the main areas of focus within the 84 AI ethics guidelines. The objectives of this paper were to first identify what were the most, and least, discussed ethical principles and values within the debate around AI use in agriculture; second, to evaluate the content of the literature, through the 11 principles; and third, to contrast the principles found in the literature not in the AI ethics guidelines (Jobin et al. 2019).

Not surprisingly, sustainability was the most discussed topic in the agricultural debate around AI, with non-maleficence, trust, and beneficence, all taking a very important emphasis within the debate, as these are often discussed in agricultural literature. Sustainability is such a significant theme in the agricultural sector, and debates around the use of AI are no exception. Perhaps, the AI ethics field as a whole can learn from this, as it was shown that there is a much lower level of discussion on topics of the environment, sustainability, and the natural world, within the AI ethics guidelines observed.

The least discussed principles within the agricultural AI literature were transparency, dignity, and solidarity. It was noted that solidarity and dignity were also not often discussed within AI ethics guidelines, as they are not always fundamentally significant for all applications and domains of AI, thus, received less attention as say privacy or justice. This is not to say that they are not important areas that require further evaluation and study, but perhaps, they may be a little less relevant for the agricultural sector as the health sector, for example.

What was concerning was the very low level of analysis of transparency within the agricultural AI literature, as this is seen as the most often principle discussed within the AI ethics guidelines. Transparency is a fundamental principle for the use and integration of AI, and often goes hand in hand with building trustworthy relationships. Thus, it was surprising to see such a minimal treatment of the topic within the agricultural AI field, particularly because trust is seen as such a significant principle to uphold. Further research should be conducted to map, analyse, and discuss how transparency can, and should, develop within agricultural AI research and development.

In addition, there were a number of topics discussed within the agricultural AI literature that was not explicitly covered within the 11 overarching ethical principles, for example, employment, the impact of AI on the non-human world, and data ownership/control. While the latter is not unique to AI, it is a growing concern in relation to any digital technology that retrieves, stores, and uses, data. Farmers are concerned about who has access to their data, who owns it, portability, and control over how it is used. There is certainly great room for the agricultural AI literature to benefit from the growing body of work underway on agricultural data-sharing (Bronson and Knezevic 2016; Carolan 2017; 
ECPA 2018; Farm Data Code of Practice Authority 2014; Fleming et al. 2018; Sonka 2016; Sonka and Cheng 2015; van der Burg et al. 2020; Wiseman et al. 2019).

Issues around employment and AI are important, and there is the concern that AI may make many people's professions redundant in the future. In agriculture, this is very concerning for many. However, the importance of employment was not fully captured within the AI ethics guidelines, as there was no explicit reference to it within the overarching principles or the subsequent coding. This may be more to do with the fact that issues of unemployment and job loss are not explicitly ethical or social issues, but are a myriad of economic, legal, and societal issues, as well. However, the impact of AI on employment certainly raises explicit social and ethical issues around justice, fairness, power, and equality, and should be given adequate treatment in agricultural $\mathrm{AI}$ and $\mathrm{AI}$ ethics.

Overall, this paper identified the newness and innovativeness of research being conducted on the social and ethical impacts of AI use in the agricultural sector (all of the articles were published within the past three years). This paper mapped what this selection of papers focused on, areas that have received more or less attention than others, and gaps where further research should be conducted. The aim of this paper is to provide a roadmap of how agricultural AI literature has developed, contrasting it with the scope of AI ethics guidelines, and areas worthy of future research.

Funding This research received funding from the Wageningen Economic Research Management Team.

\section{Declarations}

Conflict of interest The authors declare that they have no conflicting interests.

Open Access This article is licensed under a Creative Commons Attribution 4.0 International License, which permits use, sharing, adaptation, distribution and reproduction in any medium or format, as long as you give appropriate credit to the original author(s) and the source, provide a link to the Creative Commons licence, and indicate if changes were made. The images or other third party material in this article are included in the article's Creative Commons licence, unless indicated otherwise in a credit line to the material. If material is not included in the article's Creative Commons licence and your intended use is not permitted by statutory regulation or exceeds the permitted use, you will need to obtain permission directly from the copyright holder. To view a copy of this licence, visit http://creativecommons.org/licenses/by/4.0/.

\section{References}

Aggarwal N, Singh D (2021) Technology assisted farming: implications of IoT and AI. In: IOP conference series: materials science and engineering. IOP Publishing, p 012080.
Albiero D (2019) Agricultural robotics: a promising challenge. Curr Agric Res J 7:1

Anderson, J., Rainie, L., Luchsinger, A., 2018. Artificial Intelligence and the Future of Humans. Pew Res. Cent. Dec.

Aronson J (1995) A pragmatic view of thematic analysis. Qual Rep $2: 1-3$

Bekken G (2019) The algorithmic governance of data driven-processing employment: evidence-based management practices, artificial intelligence recruiting software, and automated hiring decisions Psychosociological Issues. Hum Resour Manag 7:25-30. https:// doi.org/10.22381/pihrm7220194

Belk R (2020) Ethical issues in service robotics and artificial intelligence. Serv Ind J 2:1-17

Beyan T, Felzmann H, Ryan MJ, Lang N, Beyan O (2015) D1.2 ethics framework: managing active and healthy aging with use of caring service robots

Birhane A, van Dijk J (2020) Robot rights? Let's talk about human welfare instead. In: Proceedings of the AAAI/ACM Conference on AI, Ethics, and Society. pp. 207-213.

Bogomolov A, Nevezhin V, Larionova M, Piskun E, (2021) Review of digital technologies in agriculture as a factor that removes the growth limits to human civilization. In: E3S Web of Conferences. EDP Sciences, p. 01074

Bossman J (2016) Top 9 ethical issues in artificial intelligence [WWW Document]. World Econ. Forum. URL https://www.weforum.org/ agenda/2016/10/top-10-ethical-issues-in-artificial-intelligence/ (accessed 5.10.19).

Braun V, Clarke V (2006) Using thematic analysis in psychology. Qual Res Psychol 3:77-101

Brewer S, Pearson S, Maull R, Godsiff P, Frey JG, Zisman A, Parr G, McMillan A, Cameron S, Blackmore H, Manning L, Bidaut L (2021) A trust framework for digital food systems. Nat Food 2:543-545. https://doi.org/10.1038/s43016-021-00346-1

Bronson K (2019) Looking through a responsible innovation lens at uneven engagements with digital farming. NJAS-Wagening J Life Sci 90:100294

Bronson K, Knezevic I (2016) Big data in food and agriculture. Big Data Soc 3:2053951716648174

Camaréna S (2020) Artificial intelligence in the design of transition to sustainable food systems. J Clean Prod 2:122574

Carolan MS (2006) Social change and the adoption and adaptation of knowledge claims: Whose truth do you trust in regard to sustainable agriculture? Agric Hum Values 23:325-339

Carolan M (2017) Publicising food: big data, precision agriculture, and co-experimental techniques of addition. Sociol Rural 57:135-154

Carolan M (2020) Automated agrifood futures: robotics, labor and the distributive politics of digital agriculture. J Peasant Stud 47:184-207

CNIL (2017) How can humans keep the upper hand? Report on the ethical matters raised by algorithms and artificial intelligence [WWW Document]. URL https://www.cnil.fr/en/how-can-humans-keepupper-hand-report-ethical-matters-raised-algorithms-and-artif icial-intelligence (accessed 5.14.19).

Donhauser J, van Wynsberghe A, Bearden A (2020) Steps toward an ethics of environmental robotics. Philos Technol 2:1-18

Driessen C, Heutinck LF (2015) Cows desiring to be milked? Milking robots and the co-evolution of ethics and technology on Dutch dairy farms. Agric Hum Values 32:3-20

ECPA (2018) Code of Conduct on agricultural data sharing signing [WWW Document]. ECPA. URL https://www.ecpa.eu/media/ news/code-conduct-agricultural-data-sharing-signing (accessed 8.28.20).

European Commission (2018) Artificial Intelligence for Europe (Communication No. COM(2018) 237 final). European Commission, Brussels. 
Farm Data Code of Practice Authority (2014) New Zealand Farm Data Code of Practice.

Fleming A, Jakku E, Lim-Camacho L, Taylor B, Thorburn P (2018) Is big data for big farming or for everyone? Perceptions in the Australian grains industry. Agron Sustain Dev 38:24

Floridi L (2019) Establishing the rules for building trustworthy AI. Nat Mach Intell. https://doi.org/10.1038/s42256-019-0055-y

Frey CB, Osborne MA (2017) The future of employment: how susceptible are jobs to computerisation? Technol Forecast Soc Change 114:254-280. https://doi.org/10.1016/j.techfore.2016.08.019

Gardezi M, Stock R (2021) Growing algorithmic governmentality: interrogating the social construction of trust in precision agriculture. J Rural Stud 84:1-11. https://doi.org/10.1016/j.jrurstud. 2021.03.004

Hleg AI (2020) Assessment list for trustworthy AI (ALTAI). European Commission, Brussels

Hleg A (2019) Ethics guidelines for trustworthy AI. Retrieved from High-Level Expert Group on Artificial Intelligence (AI HLEG ....

Jakku E, Taylor B, Fleming A, Mason C, Fielke S, Sounness C, Thorburn P (2019) "If they don't tell us what they do with it, why would we trust them?" Trust, transparency and benefit-sharing in Smart Farming. NJAS-Wagening. J. Life Sci. 90:100285

Jayashankar P, Nilakanta S, Johnston WJ, Gill P, Burres R (2018) IoT adoption in agriculture: the role of trust, perceived value and risk. J Bus Ind Mark 2:2

Jobin A, Ienca M, Vayena E (2019) The global landscape of AI ethics guidelines. Nat Mach Intell 1:389-399. https://doi.org/10.1038/ s42256-019-0088-2

Klerkx L, Jakku E, Labarthe P (2019) A review of social science on digital agriculture, smart farming and agriculture 4.0: New contributions and a future research agenda NJAS-Wagening. J Life Sci 90:100315

Krishnan A, Swarna S (2020) Robotics, IoT, and AI in the automation of agricultural industry: a review. in: 2020 IEEE Bangalore Humanitarian Technology Conference (B-HTC). IEEE, pp. 1-6

Lewis D, Moorkens J (2020) A rights-based approach to trustworthy AI in social media. Soc MEDIA Soc. https://doi.org/10.1177/20563 05120954672

Malta Digital Innovation Authority (2019) Malta: Towards Trustworthy AI - Malta's Ethical AI Framework. Malta.

Marinoudi V, Sørensen CG, Pearson S, Bochtis D (2019) Robotics and labour in agriculture. A context consideration. Biosyst Eng 184:111-121. https://doi.org/10.1016/j.biosystemseng.2019.06. 013

Milton CL (2021) Risking human dignity with innovations: artificial intelligence and the future of the discipline of nursing. Nurs Sci Q 34:244-246

Pylianidis C, Osinga S, Athanasiadis IN (2021) Introducing digital twins to agriculture. Comput Electron Agric 184:105942

Rodzalan SA, Yin OG, Noor NNM (2020) A foresight study of artificial intelligence in the agriculture sector in Malaysia. J Crit Rev 7:1339-1346

Rose DC, Chilvers J (2018) Agriculture 4.0: Broadening responsible innovation in an era of smart farming. Front. Sustain. Food Syst. 2:87

Rose DC, Lyon J, de Boon A, Hanheide M, Pearson S (2021) Responsible development of autonomous robotics in agriculture. Nat Food 2:306-309

Ruiz-Real JL, Uribe-Toril J, Torres Arriaza JA, de Pablo Valenciano J (2020) A look at the past, present and future research trends of artificial intelligence in agriculture. Agronomy 10:1839

Russell S (2021) Human-Compatible Artificial Intelligence. HumanLike Machine Intelligence. Oxford University Press, Oxford, pp 3-23. https://doi.org/10.1093/oso/9780198862536.003.0001
Ryan M (2019) Ethics of using AI and big data in agriculture: the case of a large agriculture multinational. ORBIT J 2:2

Ryan M (2020) Agricultural big data analytics and the ethics of power. J Agric Environ Ethics 33:49-69

Ryan M, Stahl BC (2020) Artificial intelligence ethics guidelines for developers and users: clarifying their content and normative implications. J Inf Commun Ethics Soc 2:2

Ryan M, van der Burg S, Bogaardt M-J (2021) Identifying key ethical debates for autonomous robots in agri-food: a research agenda. AI Ethics. https://doi.org/10.1007/s43681-021-00104-w

Shamshiri R, Weltzien C, Hameed IA, Yule I, Grift T, Balasundram SK, Pitonakova L, Ahmad D, Chowdhary G (2018) Research and development in agricultural robotics: a perspective of digital farming.

Sonka S (2016) Big data: fueling the next evolution of agricultural innovation. J Innov Manag 4:114-136

Sonka S, Cheng Y-T (2015) Big Data in Farming: Why Matters! Farmdoc Dly. 5:2

Sparrow R, Howard M (2020) Robots in agriculture: prospects, impacts, ethics, and policy. Precis Agric 3:1-16

Stock R, Gardezi M (2021) Make bloom and let wither: Biopolitics of precision agriculture at the dawn of surveillance capitalism. Geoforum 122:193-203

van der Burg S, Wiseman L, Krkeljas J (2020) Trust in farm data sharing: reflections on the EU code of conduct for agricultural data sharing. Ethics Inf Technol 2:1-14

van Wynsberghe A (2021) Sustainable AI: AI for sustainability and the sustainability of AI. AI Ethics 2:1-6

van Wynsberghe A, Donhauser J (2018) The dawning of the ethics of environmental robots. Sci Eng Ethics 24:1777-1800

Vasconez JP, Kantor GA, Auat Cheein FA (2019) Human-robot interaction in agriculture: a survey and current challenges. Biosyst Eng 179:35-48. https://doi.org/10.1016/j.biosystemseng.2018.12.005

Vik J, Stræte EP, Hansen BG, Nærland T (2019) The political robotThe structural consequences of automated milking systems (AMS) in Norway. NJAS-Wagening. J Life Sci 90:100305

Vincent J (2019) Forty percent of “AI startups” in Europe don't actually use AI, claims report [WWW Document]. The Verge. URL https://www.theverge.com/2019/3/5/18251326/ai-startups-europefake-40-percent-mmc-report (accessed 7.14.21).

Wang T, Xu X, Wang C, Li Z, Li D (2021) From smart farming towards unmanned farms: a new mode of agricultural production. Agriculture 11:145

Wiseman L, Sanderson J (2018) Legal and trust issues in Australian agriculture. Presented at the 40th Annual conference australian society of sugar cane technologists. ASSCT 2018:1-9

Wiseman L, Sanderson J, Zhang A, Jakku E (2019) Farmers and their data: an examination of farmers' reluctance to share their data through the lens of the laws impacting smart farming NJASWagening. J Life Sci. https://doi.org/10.1016/j.njas.2019.04.007

Ministry of Economic Affairs and Employment (2018) Work in the age of artificial intelligence.

Zardiashvili L, Fosch-Villaronga E (2020) “Oh, dignity too?" said the robot: human dignity as the basis for the governance of robotics. Minds Mach 2:1-23

Zicari RV (2021) Z-inspection a process to assess trustworthy AI. https://doi.org/10.1109/TTS.2021.3066209

Publisher's Note Springer Nature remains neutral with regard to jurisdictional claims in published maps and institutional affiliations. 